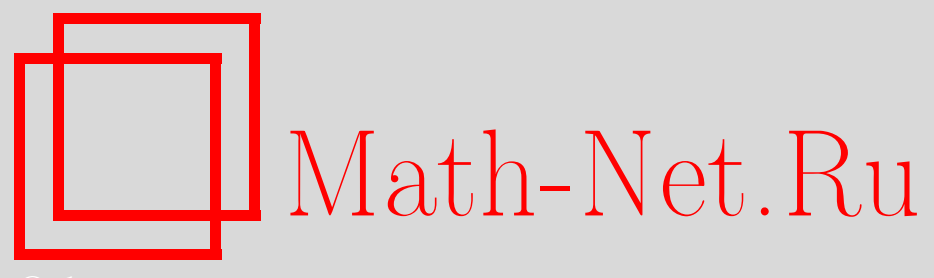

С. В. Гальцев, А. И. Шафаревич, Квантованные римановы поверхности и квазиклассические спектральные серии для несамосопряженного оператора Шредингера с периодическими коэффициентами, ТМФ, 2006, том 148, номер 2, 206-226

DOI: https://doi.org/10.4213/tmf2081

Использование Общероссийского математического портала Math-Net.Ru подразумевает, что вы прочитали и согласны с пользовательским соглашением http: //www . mathnet.ru/rus/agreement

Параметры загрузки:

IP : 54.166 .219 .16

26 апреля 2023 г., 14:13:24

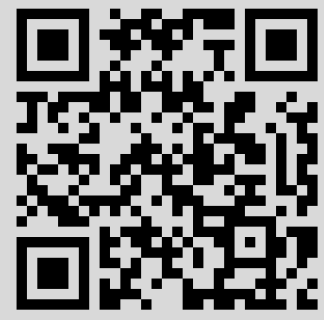




\title{
КВАНТОВАННЫЕ РИМАНОВЫ ПОВЕРХНОСТИ И КВАЗИКЛАССИЧЕСКИЕ СПЕКТРАЛЬНЫЕ СЕРИИ ДЛЯ НЕСАМОСОПРЯЖЕННОГО ОПЕРАТОРА ШРЕДИНГЕРА С ПЕРИОДИЧЕСКИМИ КОЭФФИЦИЕНТАМИ
}

\begin{abstract}
Рассматривается несамосопряженный оператор Шредингера, описывающий движение частицы в одномерном пространстве с периодическим (с вещественным периодом $T$ ) аналитическим потенциалом $i V(x)$, чисто мнимым на действительной оси. Изучается спектр этого оператора в квазиклассическом пределе. Показано, что точки спектра этого оператора асимптотически лежат на так называемом спектральном графе. Построен спектральный граф и вычислена асимптотика спектра. В фазовом пространстве можно построить риманову поверхность уравнения сохранения энергии частицы. Показано, что и спектральный граф, и асимптотика спектра вычисляются через интегралы формы $p d x(x \in \mathbb{C} / T \mathbb{Z}$ - координата, $p \in \mathbb{C}-$ импульс частицы) по базисным циклам на этой римановой поверхности. Для построения асимптотики спектра используется техника линий Стокса.
\end{abstract}

Ключевые слова: спектр, спектральный граф, несамосопряженный оператор, оператор Шредингера, линии Стокса.

\section{1. ВВЕДЕНИЕ}

Ряд вопросов, естественно возникающих в спектральной теории дифференциальных операторов, приводит к исследованию спектра оператора

$$
\mathfrak{D}=-h^{2} \frac{d^{2}}{d x^{2}}+i V(x),
$$

где $V(x)$ - периодическая целая аналитическая функция, действительная на действительной оси, с вещественным периодом $T$. В частности, оператор (1) возникает как "эталонный" оператор в теории гидродинамической устойчивости: его спектр

*Московский государственный университет, Москва, Россия. E-mail: galtsev@mccme.ru, shafar@mech.math.msu.su 
при определенных условиях похож на спектр оператора Орра-Зоммерфельда. Другой пример - спектральная задача для оператора $\varepsilon \Delta+(v(x), \nabla)$ на плоском тоpe (здесь $\left.x=\left(x_{1}, x_{2}\right) \in \mathbb{T}^{2}\right)$. Если $v(x)$ - бездивергентное поле вида $v\left(x_{1}, x_{2}\right)=$ $w\left(x_{1}\right) \partial / \partial x_{2}$, спектральная задача допускает разделение переменных: собственная функция $\varphi\left(x_{1}, x_{2}\right)$ имеет вид $e^{i m x_{2}} \psi\left(x_{1}\right)$, причем функция $\psi$ удовлетворяет спектральной задаче для оператора (1) при $V\left(x_{1}\right)=m w\left(x_{1}\right)$ и $\varepsilon=h^{2}$.

Спектральная теория несамосопряженных операторов по сравнению с самосопряженным случаем разработана значительно менее полно; как структура спектра, так и свойства спектрального разложения могут в этом случае быть весьма экзотическими, что чрезвычайно затрудняет развитие общей теории (см., например, [1]). В то же время ряд важных задач, возникающих в различных областях математики и математической физики, приводит к изучению спектров несамосопряженных операторов. Приведем несколько популярных примеров.

1. Оператор диффузии со сносом $\varepsilon \Delta+\partial_{v}(\Delta-$ оператор Лапласа-Бельтрами, $\partial_{v}$ - производная вдоль гладкого векторного поля $v$ на римановом многообразии, $\varepsilon>0$ - коэффициент диффузии), возникающий как в механике сплошных сред и кинетической теории, так и в задачах теории случайных процессов.

2. Оператор магнитной индукции $\widetilde{M}$, действующий на магнитное поле $H$ в проводящей жидкости с полем скоростей $v\left(H, v-\right.$ векторные поля в $\left.\mathbb{R}^{3}\right)$ :

$$
\widetilde{M}=\{v, H\}-\varepsilon \Delta H,
$$

где $\{\cdot, \cdot\}$ - коммутатор векторных полей, $\varepsilon>0$ - проводимость. Исследование поведения спектра этого оператора при $\varepsilon \rightarrow 0$ связано с известной проблемой магнитного динамо (см., например, [2]).

3. Оператор Орра-Зоммерфельда $Q$, возникающий в теории гидродинамической устойчивости (см., например, [3]); этот оператор действует на функцию $u(x)$ по правилу

$$
Q u=i \varepsilon\left(\frac{d^{2}}{d x^{2}}-p^{2}\right)^{2} u+p\left((v(x)-\omega)\left(\frac{d^{2}}{d x^{2}}-p^{2}\right)-v^{\prime \prime}(x)\right) u
$$

где $v(x)$ - гладкая функция (невозмущенный профиль скорости), $p$ - волновое число возмущения, $\omega$ - частота (спектральный параметр), $\varepsilon$ - коэффициент вязкости $\left(\varepsilon^{-1}\right.$ число Рейнольдса).

Отметим, что во всех приведенных примерах присутствует параметр $\varepsilon$, который во многих типичных ситуациях бывает малым, тем самым естественный вопрос состоит в изучении предельного поведения спектра при $\varepsilon \rightarrow 0$. Для самосопряженных задач такие пределы изучаются в теории квазиклассических асимптотик (см., например, [4]). В этой теории асимптотические собственные числа и собственные функции связываются с инвариантными изотропными многообразиями соответствующих классических гамильтоновых систем (как правило, определенных в $\mathbb{R}^{2 n}$ или в 
кокасательном расслоении к риманову многообразию). Собственные числа вычисляются из условий квантования Бора-Зоммерфельда-Маслова

$$
\frac{1}{2 \pi \varepsilon} \int_{\gamma}(p, d x)=m(\gamma)+l(\gamma),
$$

где $\gamma$ - произвольный цикл на изотропном многообразии, $(p, x)$ - канонические координаты в фазовом пространстве, скобка в подынтегральном выражении означает скалярное произведение $(p, d x)=p_{1} d x_{1}+\cdots+p_{n} d x_{n}, m \in \mathbb{Z}, l$ - числовая характеристика циклов, которая определяется по-разному в различных ситуациях; в частности, если изотропное многообразие лагранжево, $l$ равно четверти от индекса Маслова. Асимптотические собственные функции строятся при помощи (вещественного или комплексного) канонического оператора Маслова; отметим, что они не обязательно приближают настоящие собственные функции исходной задачи, а лишь удовлетворяют с нужной точностью спектральному уравнению. В то же время самосопряженность исходного оператора гарантирует наличие в его спектре точек, близких к асимптотическим собственным числам (решениям уравнений Бора-Зоммерфельда).

Относительно квазиклассических асимптотик спектров несамосопряженных операторов известно гораздо меньше. В частности, в работах [5] построены спектральные серии оператора $-\varepsilon \Delta+\partial_{v}$, связанные с асимптотически устойчивыми положениями равновесия, предельными циклами или инвариантными торами векторного поля $v$. (Спектральные серии, вообе говоря, определяют точки псевдоспектра (см. [6]), в то же время асимптотическая устойчивость соответствующих инвариантных множеств указывает на то, что, вероятно, эти серии приближают точные собственные числа - в точно решаемых примерах это действительно так.) В работах [7], [8] полностью исследован спектр одномерного оператора Шредингера и оператора Орра-Зоммерфельда на отрезке (отметим, что ряд утверждений об условиях квантования содержался еще в работе [9]). В этих работах, основанных на развитой в [10], [11] технике линий Стокса, было обнаружено, что в квазиклассическом пределе спектр стягивается к некоторому графу на комплексной плоскости (так называемому спектральному графу или “спектральному галстуку"), причем ребра этого графа задаются геометрическими условиями на линии Стокса (одна из этих линий должна проходить через конец отрезка, на котором рассматривается уравнение).

В настоящей работе исследуется спектр одномерного оператора Шредингера на окружности с комплексным (чисто мнимым) потенциалом. К такой задаче сводится, в частности, вычисление спектра оператора $\varepsilon \Delta+\partial_{v}$ на плоском торе в простейшем случае бездивергентного поля $v$, допускающего разделение переменных. Оказывается, точки спектра в квазиклассическом пределе могут быть вычислены из условий Бора-Зоммерфельда-Маслова, записанных на римановой поверхности; однако, в отличие от самосопряженного случая, достаточно требовать выполнения этих условий хотя бы на одном из базисных циклов этой поверхности, причем разные циклы дают 
в спектр вклад, соответствующий разным ребрам спектрального графа. Как и в работах [7], [8], спектральный граф связан с портретом линий Стокса; именно, ребра спектрального графа соответствуют перестройкам графа Стокса. Отметим, что в силу периодичности задачи таких перестроек счетное число, однако оказывается, что в действительности ребрам спектрального графа отвечает лишь несколько из них.

\section{2. ПОСТАНОВКА ЗАДАЧИ И ФОРМУЛИРОВКА РЕЗУЛЬТАТА}

В настоящей работе исследуется асимптотика спектра оператора (1). Этот оператор естественно рассматривать как неограниченный несамосопряженный оператор в $L_{2}\left(\mathbb{S}^{1}=\mathbb{R} / T \mathbb{Z}\right)$. В то же время, поскольку уравнение $(\mathfrak{D}-E) \varphi=0$ - это обыкновенное линейное дифференциальное уравнение с целыми аналитическими коэффициентами, все его решения - аналитические функции, и оператор $\mathfrak{D}$ можно рассматривать как оператор в пространстве $\mathcal{A}(\mathbb{C} / T \mathbb{Z})$ целых аналитических периодических функций. Цель настоящей работы - описание асимптотики при $h \rightarrow 0+0$ спектра оператора $\mathfrak{D}$ в частном случае $V(x)=\cos x$.

Оказывается, эта асимптотика выражается через интегралы от голоморфных форм по циклам на римановой поверхности $\Lambda$, задаваемой в $\mathbb{C}^{2} / T \mathbb{Z}$ уравнением $p^{2}+i V(x)=E, p \in \mathbb{C}, x \in \mathbb{C} / T \mathbb{Z}$. Эта (некомпактная) поверхность при $E \neq \pm i$ (в дальнейшем мы будем работать с параметром $E$, отделенным от $\pm i$ ) гомеоморфна
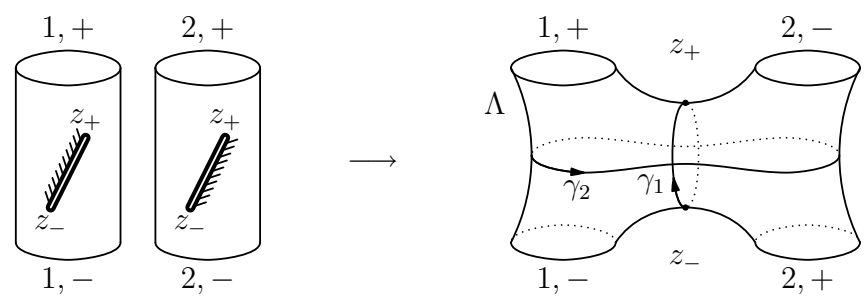

Рис. 1

сфере с четырьмя дырками и получается склейкой двух экземпляров цилиндра $\mathbb{C} / T \mathbb{Z}$ по отрезку, соединяющему нули функции $i V(x)-E$ (см. рис. 1). На рисунке символами $1,+; 2,+; 1,-; 2,-$ обозначены дырки поверхности $\Lambda$, причем 1 или 2 обозначает номер цилиндра, а "+" или “-” обозначает знак $\operatorname{Im} z$.

Следующая теорема представляет собой основной результат работы.

Теорема 1. Пусть параметр $E$ (отделенный от $\pm i$ ) таков, что на поверхности $\Lambda$ существует иикл $\gamma$, для которого

$$
\frac{1}{2 \pi h} \int_{\gamma} p d x=m+\frac{\mu}{2} .
$$


Тогда существует собственное число $\lambda$ оператора $\mathfrak{D}$, для которого $\lambda-E=O\left(h^{2}\right)$. Здесъ $\mu=0$, если цикл $\gamma$ становится стягиваемым при заклеивании некоторой дырки на $\Lambda, u \mu=1$ в противоположном случае.

ЗАмечаниЕ 1. Условия (2) похожи на условия квантования Бора-Зоммерфельда-Маслова лагранжевых многообразий в $\mathbb{R}^{2 n}$ (см., например, [4]), описывающие спектр оператора вида $H(x,-i h \partial / \partial x)$. Отметим, однако, что в теории квазиклассического квантования требуется выполнение условий квантования для всех циклов на $\Lambda$, тогда как в нашем случае достаточно выполнения условия (2) хотя бы для одного цикла.

ЗАмЕчАНИЕ 2. На поверхности $\Lambda$ имеются три независимых цикла, выберем базис циклов следующим образом. В качестве $\gamma_{1}$ и $\gamma_{2}$ возьмем циклы, обходящие две дырки на $\Lambda$, как показано на рис. 1 . В качестве $\gamma_{3}$ возьмем любой цикл, обходящий одну из дырок поверхности $\Lambda$. Условия (2) запишутся в виде

$$
\begin{aligned}
& \frac{1}{2 \pi h} \int_{\gamma_{j}} p d x=m_{j}+\frac{1}{2}, \quad j \in\{1,2\}, \\
& \frac{1}{2 \pi h} \int_{\gamma_{3}} p d x=m_{3} .
\end{aligned}
$$

Тем самым выделяется три серии собственных чисел - каждая соответствует решениям одного из трех уравнений (3). Оказывается, при $h \rightarrow 0$ собственные значения концентрируются в $O\left(h^{2}\right)$-окрестности графа на комплексной плоскости. Ребра этого графа удовлетворяют уравнениям

$$
\frac{1}{2 \pi h} \operatorname{Re} \int_{\gamma_{j}} p d x=0 .
$$

Назовем этот граф предельным спектральным графом оператора $\mathfrak{D}$. Вне окрестностей точек $\pm i$ предельный спектральный граф изображен на рис. 2.

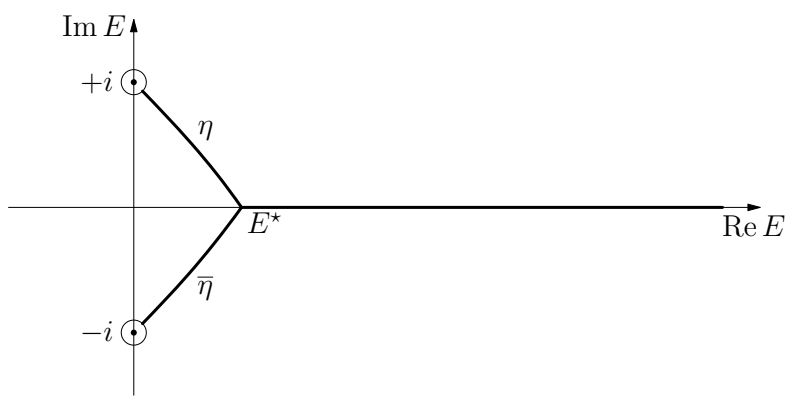

Рис. 2

ЗАмечАниЕ 3. Предельный спектральный граф связан с графом Стокса уравнения $(\mathfrak{D}-E) \varphi=0$. Напомним, что граф Стокса определяется следующим образом. 
Обозначим через $z_{ \pm}(E)$ решения уравнения $i V(x)=E$ на цилиндре; они называются точками поворота. Для каждой из этих точек рассмотрим три выходящие из нее кривые, заданные уравнением на переменную z

$$
\operatorname{Re} \int_{z_{ \pm}}^{z} \sqrt{E-i V(w)} d w=0 .
$$

Эти линии называются линиями Стокса, их объединение образует граф Стокса. Оказывается, предельный спектральный граф оператора (1) обладает следующим свойством: если $E$ принадлежит спектральному графу, соответствующий граф Стокса содержит конечную линию Стокса. Другими словами, в этом случае линия Стокса, выходящая из одной точки поворота, попадает в нее же или в другую.

Существование конечной линии Стокса - необходимое условие, однако оно не является достаточным. Дело в том, что есть топологическая конфигурация графа Стокса, имеющая конечную линию Стокса, но не вносящая вклад в спектр. Кривых в $E$-плоскости, на которых реализуются графы Стокса, содержащие конечную линию Стокса, имеется счетное число. Они показаны на рис. 3, где они занумерованы

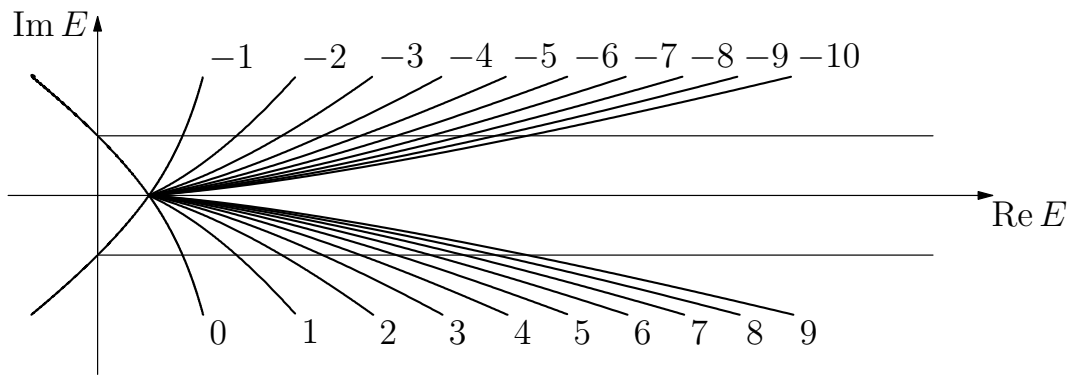

Рис. 3

числом $k$ : на $k$-й кривой реализуется граф Стокса, в котором точки $z_{-}$и $z_{+}-2 \pi k$ соединены конечной линией Стокса. На рис. 3 также показаны границы числового образа оператора $\mathfrak{D}$, который является полуполосой $[0,+\infty)+i[-1,1]$.

План доказательства теоремы 1 следующий. Для того чтобы найти спектр оператора (1), воспользуемся приближением Вентцеля-Крамерса-Бриллюэна (ВКБ). Для этого прежде всего выясним, как может выглядеть граф Стокса для интересующего нас потенциала $i V(z)=i \cos z$. Этот граф зависит от параметра $E$ и при его изменении может перестраиваться. Другими словами, в зависимости от значения $E$ возможны топологически различные случаи реализации графа Стокса. После того как все возможные случаи будут найдены, в каждом из них вычислим матрицу монодромии (сдвига аргумента на период) оператора (1). Эта матрица позволит найти, при каких значениях $E$ у исследуемого оператора есть периодическое решение. Точнее, из условия на матрицу монодромии мы получим условие на спектр оператора (1). 


\section{3. ТОЧКИ ПОВОРОТА}

Прежде всего отметим следующее (доказываемое прямой проверкой) свойство дискретного спектра оператора $\mathfrak{D}$ в рассматриваемом случае.

ЛЕмма 1. При любом $h$ собственные значения оператора $\mathfrak{D}$ с потенииалом $i V(z)=i \cos z$ симметричны относительно оси $\operatorname{Re} E$.

Конструкция ВКБ-асимптотик начинается с нахождения точек поворота, т.е. с решения уравнения $i V(z)=E$. Эти точки расположены на комплексной плоскости периодически с периодом $T$.

Лемма 2. Точки поворота являются непрерывными функциями параметра Е. Они задаются формулой

$$
z_{ \pm}(E)=-i \operatorname{Ln}\left(-i E \pm \sqrt{-E^{2}-1}\right) .
$$

ДокАЗАтЕЛЬСтво. Множество решений уравнения $i \cos z=E$ может быть задано как множество точек $-i \operatorname{Arch}(-i E)=-i \operatorname{Ln}\left(-i E \pm \sqrt{-E^{2}-1}\right)$. При этом аргумент Ln не обращается в нуль или бесконечность при любом $E \in \mathbb{C}$. Следовательно, решения уравнения $i \cos z=E$ аналитичны на всей комплексной плоскости, а значит, они непрерывны.

Введем обозначение

$$
\xi\left(z_{1}, z_{2}\right)=\int_{z_{1}}^{z_{2}} \sqrt{i \cos z-E} d z .
$$

В дальнейшем нам придется подставлять точки поворота в качестве пределов в этот интеграл. Поэтому мы будем считать, что $z_{1}$ и $z_{2}$ зависят от параметра $E$. Легко проверяется следующее утверждение.

ЛЕмма 3. Функция $\xi\left(z_{1}, z_{2}\right)$ непрерывно дифферениируема по параметру $E \in$ $\mathbb{C} \backslash(U(i) \cup U(-i))$, где $U( \pm i)$ - некоторые окрестности точек $\pm i$.

\section{4. ЛИНИИ СТОКСА}

В зависимости от значения $E$ взаимное расположение линий Стокса, т.е. линий $\gamma$, выходящих из точек поворота $z_{ \pm}$и удовлетворяющих условию

$$
\left.\operatorname{Re} \xi\left(z_{ \pm}, z\right)\right|_{\gamma}=0, \quad \xi\left(z_{ \pm}, z\right)=\int_{z_{ \pm}}^{z} \sqrt{i V(w)-E} d w
$$

меняется. Выясним возможное взаимное расположение линий Стокса для функции $V(z)=\cos z$ при различных значениях $E$ на основе следующих свойств линий Стокса в случае целой функции $V(z)$ (см. [11]):

1) линия Стокса начинается в точке поворота и заканчивается либо в точке поворота, либо в бесконечности;

2) линия Стокса не может содержать точку поворота внутри себя; 
3) линия Стокса не может пересекать себя или другую линию Стокса;

4) из точки поворота кратности $d$ выходят $d+2$ линии Стокса;

$5)$ граф линий Стокса (на плоскости $\mathbb{C}$ ) не может содержать топологическую окружность;

6) линии Стокса разбивают плоскость $\mathbb{C}$ на области типа полуплоскости и полосы (т.е. на области, имеющие в качестве границы одну топологическую прямую или дизъюнктное объединение двух топологических прямых).

Случай кратных точек поворота $(E= \pm i)$ рассматривать не будем.

Отметим, что поскольку функция $V(z)$ периодична с периодом $T$, то и граф линий Стокса периодичен с тем же периодом. Кроме того, так как функция косинус четна $(\cos (-z)=\cos z)$, то в случае $V(z)=\cos z$ граф линий Стокса симметричен относительно нуля. Также отметим, что бесконечностей на цилиндре $\mathbb{C} / T \mathbb{Z}$ две, а именно $\pm i T \infty$ (при $T \in \mathbb{R}: \pm i \infty)$.

В дальнейшем мы будем выписывать матрицы монодромии, которые строятся по-разному для топологически различных графов Стокса и одинаково (по модулю конкретных значений констант) для топологически одинаковых графов Стокса. Поэтому топологически эквивалентные графы Стокса мы будем относить к одному случаю.

ТеОрема 2. В случае $i V(z)=i \cos z$ имеется пять топологически различных случаев взачмного расположения линий Стокса, приведенных на рис. 4.

1.

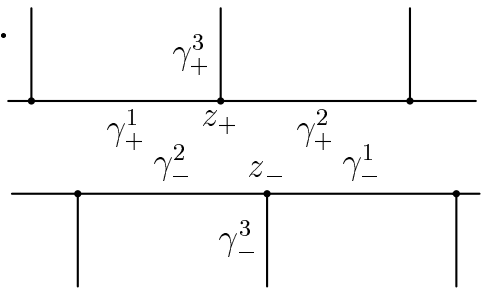

2.

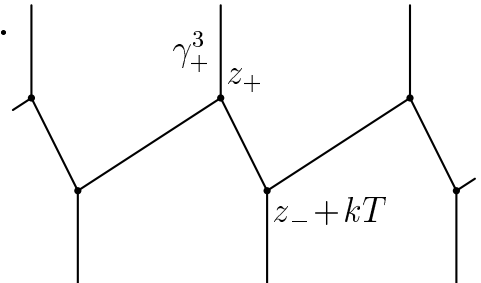

3.

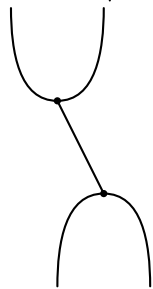

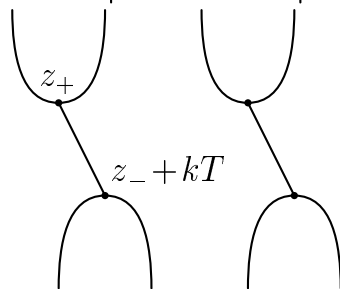

5 .

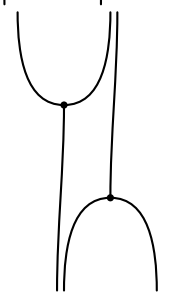

4.

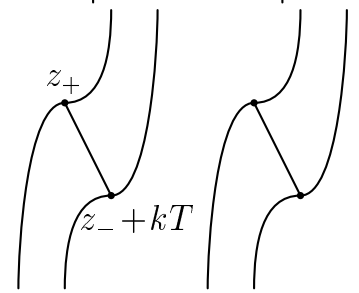

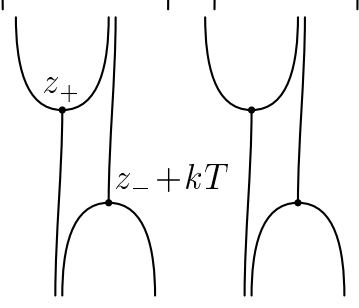

Рис. 4 
ДокАЗАТЕЛЬСтво следует из приведенных выше свойств 1-6 линий Стокса. На рис. $4 k \in \mathbb{Z}$. Во всех вариантах графов Стокса точка поворота $z_{+}$может быть соединена только с точкой поворота $z_{+}+T$ или $z_{-}+k T$.

ЛЕмма 4. Если точки $z_{+}$и $z_{+}+2 \pi$ соединены в графе Стокса (т.е. соединены криволинейной ломаной из линий (токса), то

$$
I(E)=\operatorname{Re} \int_{0}^{2 \pi} \sqrt{i \cos x-E} d x=0 .
$$

Для доказательства этой леммы достаточно в качестве пути интегрирования взять ломаную из линий Стокса.

Лемма 5. Если точки $z_{+}$и $z_{-}$соединены в графе Стокса, то верно равенство

$$
J(E)=\operatorname{Re} \int_{z_{-}(E)}^{z_{+}(E)} \sqrt{i \cos x-E} d x=\xi\left(z_{-}, z_{+}\right)=0 .
$$

Если соединены точки $z_{+}-2 \pi k u z_{-}$, то $\xi\left(z_{-}, z_{+}-2 \pi k\right)=0$. В частности, при $k=-1$ имеем $J(\bar{E})=0$.

Эта лемма аналогична предыдущей (с учетом леммы 11, см. приложение).

Лемма 6. Случай 1 топологической конфигурации графа Стокса может реализоваться, только если $E \in(0,+\infty)$. Случай 2 топологической конфигурации графа Стокса может реализоваться только при $E=E^{\star}$.

ДокАЗАтЕЛЬСтво. Эти два случая удовлетворяют условию леммы 4. Объединяя лемму 4 и лемму 12 (см. приложение), приходим к выводу, что эти случаи могут реализоваться только при $E \in(0,+\infty)$.

Для топологического случая 2 дополнительно отметим, что он удовлетворяет еще и условию леммы 5, а значит, и леммы 13 (см. приложение). Следовательно, этот случай может реализоваться только при $E=E^{\star}$.

Лемма 7. Топологические случаи 3 и 4 могут реализоватъся только на аналитических кривых в Е-плоскости, на каждой из которых выполняется условие

$$
\operatorname{Re} \int_{z_{-}(E)}^{z_{+}(E)-2 \pi k} \sqrt{i \cos x-E} d x=0
$$

для некоторого $k \in \mathbb{Z}$. Все эти кривые (обозначим их $\eta_{k}$ ) проходят через точку $E^{\star}$ u пересекают луч $(0,+\infty)$ только в этой точке. Кривые $\eta_{k}$ и $\eta_{-k-1}$ симметричны друг другу относительно оси $\operatorname{Re} E: \eta_{-k-1}=\overline{\eta_{k}}$. Кривая $\eta_{0}$ проходит через точку $i$.

ДокАЗАтЕльство. Возможность реализации топологических случаев 3 и 4 только на кривых $\eta_{k}$ следует из леммы 5 . Симметрия относительно оси $\operatorname{Re} E$ следует из леммы 11 . Все кривые $\eta_{k}$ проходят через точку $E^{\star}$, так как в ней имеем одновременно $\xi\left(z_{-}, z_{+}\right)=0$ и $\xi(0,2 \pi)=0$. Согласно леммам 5 и 13 кривая $\eta_{0}$ пересекает луч $(0,+\infty)$ только в точке $E^{\star}$. При $E=i$ точки $z_{+}(E)$ и $z_{-}(E)$ совпадают, и в силу непрерывности $\xi\left(z_{-}, z_{+}\right)$имеем $\xi\left(z_{-}, z_{+}\right) \rightarrow \xi\left(z_{-}(i), z_{+}(i)\right)=0$. Следовательно, кривая $\eta_{0}$ проходит через точку $i$. 


\section{5. РЕАЛИЗУЕМОСТЬ ТОПОЛОГИЧЕСКИХ СЛУЧАЕВ}

Как мы знаем, спектр принадлежит полуполосе $[0,+\infty)+i[-1,1]$. Нам придется исключить из нее окрестности точек $\pm i$. Оставшееся множество разбивается на подмножества, на которых достигаются различные топологические случаи, описанные в теореме 2. Нашей целью является построение этого разбиения. Прежде всего докажем, что топологический случай 4 достигается только при $k=0$ и $k=-1$.

Лемма 8. В окрестности точки $E^{\star}$ топологический случай 4 достигается только при $k=0$ и $k=-1$.

ДокАЗАтЕЛЬСтво. В окрестности точки $E^{\star}$ по непрерывности функции $\xi$ линии уровня $\left.\operatorname{Re} \xi\right|_{E \in U\left(E^{\star}\right)}=$ const близки к линиям уровня $\left.\operatorname{Re} \xi\right|_{E=E^{\star}}=$ const. Отметим, что поле $\operatorname{grad} \operatorname{Im} \xi\left(E, z_{0}, z\right)=(\partial \operatorname{Im} \xi / \partial \operatorname{Re} z, \partial \operatorname{Im} \xi / \partial \operatorname{Im} z)$ является касательным для линий уровня $\operatorname{Re} \xi\left(z_{0}, z\right)=$ const при фиксированных $E$ и $z_{0}$.

При $E=E^{\star}$ в графе Стокса имеется ломаная $Г$ из линий Стокса, ведущая из точки $z_{+}\left(E^{\star}\right)-T$ в точку $z_{-}\left(E^{\star}\right)+k T$. Обозначим отрезок $\left[z_{+}\left(E^{\star}\right)-T, z_{-}\left(E^{\star}\right)\right]$ этой ломаной через $\Gamma_{0}$, отрезок $\Gamma_{0}+T=\left[z_{+}\left(E^{\star}\right), z_{-}\left(E^{\star}\right)+T\right]-$ через $\Gamma_{0}^{\prime}$, а отрезок $\left[z_{-}\left(E^{\star}\right), z_{+}\left(E^{\star}\right)\right]$ - через $\Gamma_{1}$. Отрезки $\Gamma_{0}, \Gamma_{0}^{\prime}$ и $\Gamma_{1}$ суть линии Стокса при $E=E^{\star}$ (см. рис. 5 , построенный для случая $k=1$ ).

Предположим, что в окрестности точки $E^{\star}$ существует такое $E_{0}$, что при $E=$ $E_{0}$ имеется конечная линия Стокса, не однозначно проецирующаяся на основание цилиндра $\mathbb{S}^{1}=\mathbb{R} / T \mathbb{Z}$ ("обматывающая" цилиндр $\mathbb{C} / T \mathbb{Z}$ ). Обозначим ее $\gamma_{2}$. Для определенности будем считать, что она начинается в точке $z_{+}\left(E_{0}\right)-T$ и идет "вправо" (см. рис. 5), т.е. она близка к $\Gamma_{0}$ в окрестности своей начальной точки $z_{+}\left(E_{0}\right)-T$

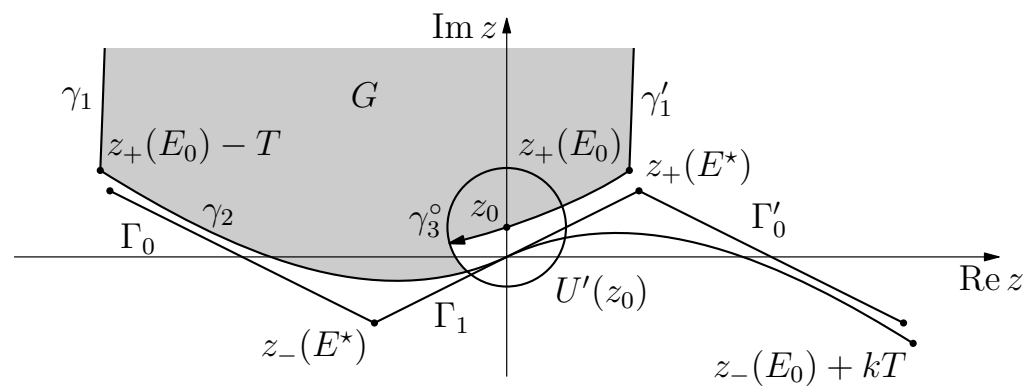

Рис. 5

(здесь и далее мы берем достаточно большую окрестность, чтобы отрезок $\Gamma_{0}$ с ней пересекался; это то же самое, что говорить о близости начальных векторов $\gamma_{2}$ и $\left.\Gamma_{0}\right)$. Как мы знаем (см. теорему 2), эта линия не может заканчиваться в точке $z_{+}\left(E_{0}\right)+k T$ при $k \in \mathbb{N}$. Обозначим точку, в которой она заканчивается, через $z_{-}\left(E_{0}\right)+k T$ для некоторого $k \in \mathbb{N}$.

Кроме того, из точки $z_{+}\left(E_{0}\right)-T$ и точки $z_{+}\left(E_{0}\right)$ должны выходить линии Стокса $\gamma_{1}$ и $\gamma_{1}^{\prime}=\gamma_{1}+T$ в направлении $+i T \infty$ на цилиндре $\mathbb{C} / T \mathbb{Z}$, так как они должны идти 
в бесконечность (по теореме 2), а кроме того, они должны быть близки к уходящим в $+i T \infty$ из точек $z_{+}\left(E^{\star}\right)-T$ и $z_{+}\left(E^{\star}\right)$ линиям Стокса при $E=E^{\star}$. Из точки $z_{+}\left(E_{0}\right)$ по периодичности должна также выходить линия Стокса $\gamma_{2}+T$, идущая вправо на рис. 5 (т.е. близкая к $\Gamma_{0}^{\prime}$ в окрестности своей начальной точки). Остается только понять, куда идет третья линия Стокса $\gamma_{3}$, выходящая из точки $z_{+}\left(E_{0}\right)$ влево (т.е. в окрестности точки $z_{+}\left(E_{0}\right)$ близкая к $\left.\Gamma_{1}\right)$. Согласно теореме 2 эта линия Стокса уходит в одну из бесконечностей. Если она уходит в $+i T \infty$, получаем топологический случай 3 , если же она уходит в $-i T \infty$, получаем топологический случай 4 . Мы покажем, что она уходит в $+i T \infty$. Тогда в окрестности $E^{\star}$ топологический случай 4 не реализуется при $k \in \mathbb{N}$, а реализуется только на двух кривых (соответствующих $k=0$ и $k=-1$ ), так как при этих $k$ его конечная линия Стокса однозначно проецируется на $\mathbb{R} / T \mathbb{Z}$ (не “обматывает" цилиндр $\mathbb{C} / T \mathbb{Z}$ ).

Линия Стокса $\gamma_{2}$ должна проходить ниже точки $z_{+}\left(E_{0}\right)$, иначе она пересечет линию Стокса $\gamma_{1}^{\prime}$. Выбрав достаточно малую окрестность $U\left(E^{\star}\right)$, можно добиться того, чтобы кривая $\gamma_{2}$ проходила в достаточно малой окрестности точки $z_{+}\left(E_{0}\right)$. Тогда начальный отрезок (как минимум до окрестности точки $z_{-}\left(E^{\star}\right)$ ) линии Стокса $\gamma_{3}$ лежит в достаточно малой окрестности линии Стокса $\gamma_{2}$. Выберем точку $z_{0}$ на линии $\gamma_{3}$ вдали от точек поворота, например точку, в которой линия $\gamma_{3}$ пересекает ось $\operatorname{Im} z$. Можно считать, что окрестность $U\left(E^{\star}\right)$ была выбрана таким образом, что в некоторой окрестности $U^{\prime}\left(z_{0}\right)$, пересекающейся с $\gamma_{2}$, поле $\left.\operatorname{grad} \operatorname{Im} \xi\left(z_{+}\left(E_{0}\right), z\right)\right|_{E=E_{0}}$ отклонялось бы от своего направления в точке $z_{0}$ на угол, не превышающий (по модулю) $\alpha$, где $\alpha \in(0, \pi / 2)$. Обозначим через $\gamma_{3}^{\circ}$ начальный отрезок $\gamma_{3}$, начинающийся в точке $z_{+}\left(E_{0}\right)$ и заканчивающийся в точке, в которой $\gamma_{3}$ выходит из окрестности $U^{\prime}\left(z_{0}\right)$.

Предположим, что линия $\gamma_{3}$ уходит в $-i T \infty$. Тогда она должна выходить из области $G$, ограниченной линиями Стокса $\gamma_{2}, \gamma_{1}, \gamma_{1}^{\prime}$, начальным отрезком $\gamma_{3}^{\circ}$ и частью границы $\partial U^{\prime}\left(z_{0}\right)$, так как эта область не содержит $-i T \infty$ (она содержит только $+i T \infty)$. То есть линия $\gamma_{3}$ должна пересекать границу $\partial G$ области $G$ (а значит, линия $\gamma_{3}$ должна пересекать или $\gamma_{2}$, или $\gamma_{1}$, или $\gamma_{1}^{\prime}$, или $\gamma_{3}^{\circ}$, или $\left.\partial U^{\prime}\left(z_{0}\right)\right)$. Но линия Стокса не может пересекать себя или другую линию Стокса нигде, кроме точки поворота, а $\gamma_{3}$, как мы уже выяснили, уходит в бесконечность и, значит, не может входить в точку $z_{+}-T$ или точку $z_{+}$. Таким образом, $\gamma_{3}$ может выйти из области $G$, только если пересечет границу $\partial U^{\prime}\left(z_{0}\right)$. При этом для того чтобы выйти из $G$, линия Стокса $\gamma_{3}$ должна пройти через $U^{\prime}\left(z_{0}\right)$ в направлении, близком к противоположному тому направлению, в котором она проходит через эту окрестность первый раз (когда проходит через точку $\left.z_{0}\right)$. Так как в окрестности $U^{\prime}\left(z_{0}\right)$ модуль угла $\alpha$ между $\left.\operatorname{grad} \operatorname{Im} \xi\left(z_{+}\left(E_{0}\right), z\right)\right|_{E=E_{0}}$ и $\left.\operatorname{grad} \operatorname{Im} \xi\right|_{z=z_{0}}$ не превосходит $\pi / 2$, получаем, что при втором проходе линии $\gamma_{3}$ через окрестность $U^{\prime}\left(z_{0}\right)$ направление изменения $\operatorname{Im} \xi$ вдоль нее должно быть противоположным направлению изменения $\operatorname{Im} \xi$ вдоль нее же во время первого прохода через окрестность $U^{\prime}\left(z_{0}\right)$.

Остается заметить только, что поскольку $\operatorname{Im} \xi$ строго монотонно возрастает вдоль линии Стокса (см. [11]), то мы получили противоречие. Таким образом, линия $\gamma_{3}$ не 
может уйти в $-i T \infty$, а значит, она уходит в $+i T \infty$. Это, в свою очередь, означает, что топологический случай 4 в окрестности точки $E^{\star}$ реализуется только на двух (сопряженных) кривых, задающихся в $E$-плоскости двумя уравнениями $\operatorname{Re} \int_{z_{-}(E)}^{z_{+}(E)} \sqrt{i \cos z-E} d z=0$ и $\operatorname{Re} \int_{z_{-}(E)}^{z_{+}(E)-2 \pi} \sqrt{i \cos z-E} d z=0$.

Лемма 9. На кривых $\eta_{k}$ и на луче $(0,+\infty)$ смена топологического вида графа Стокса может происходить только в точке $E^{\star}$.

Доказательство. Так как $z_{ \pm}$и линии Стокса непрерывны по параметру $E$, а различные топологические случаи определяются попаданием или непопаданием линий Стокса в различные точки поворота, то:

1) топологический случай 5 (случай общего положения) реализуется на открытом подмножестве $E$-плоскости;

2) топологические случаи 3 и 4 реализуются на открытых подмножествах кривых, для которых $\operatorname{Re} \xi\left(z_{-}+k T, z_{+}\right)=0$ (при каждом фиксированном $\left.k \in \mathbb{Z}\right)$;

3) топологический случай 1 достигается на открытом подмножестве множества, на котором верно $\operatorname{Re} \xi\left(z_{+}, z_{+}+T\right)=0$ (как мы знаем, последнее множество луч $(0,+\infty))$;

4) топологический случай 2 достигается на открытом подмножестве множества, на котором одновременно $\operatorname{Re} \xi\left(z_{-}, z_{+}\right)=0$ и $\operatorname{Re} \xi\left(z_{-}+T, z_{+}\right)=0$ (как мы знаем, указанные множество и его подмножество - это $\left.\left\{E^{\star}\right\}\right)$.

Таким образом, вдоль кривых в $E$-плоскости, на которых $\operatorname{Re} \xi\left(z_{-}+k T, z_{+}\right)=0$, топологический вид графа Стокса может измениться только в точке $E^{\star}$. Действительно, рассмотрим любую точку $E_{0} \in \eta_{k} \backslash\left\{E^{\star}\right\}$. Связная окрестность этой точки на кривой $U\left(E_{0}\right) \cap \eta_{k} \backslash\left\{E^{\star}\right\}$ разбивается на три множества $U_{j}$, на каждом из которых достигается топологический случай $j \in\{3,4,5\}$ (согласно леммам 6 и 7 топологические случаи 1 и 2 не достигаются в достаточно малой окрестности любой точки $\left.E_{0} \in \eta_{k} \backslash\left\{E^{\star}\right\}\right)$. Каждое из множеств $U_{j}$ является открытым на $\eta_{k}\left(U_{5}\right.$ является открытым на $\eta_{k}$, так как является пересечением открытого в $\mathbb{C}$ множества с $\left.\eta_{k}\right)$. Другими словами, мы имеем разбиение множества $U\left(E_{0}\right) \cap \eta_{k}$ на три открытые в нем компоненты: $U\left(E_{0}\right)=U_{3} \cup U_{4} \cup U_{5}$. Так как множество $U\left(E_{0}\right) \cap \eta_{k} \backslash\left\{E^{\star}\right\}$ связно, то две из этих трех компонент должны быть пусты. Другими словами, в $U\left(E_{0}\right) \cap \eta_{k} \backslash\left\{E^{\star}\right\}$ реализуется только один топологический случай, а значит, вдоль кривой $\eta_{k}$ смена топологического случая может происходить только в точке $E^{\star}$.

Аналогично доказывается утверждение о том, что на луче $(0,+\infty)$ смена топологического случая может произойти только в точке $E^{\star}$.

СлЕДСТВИЕ 1. На интервале $\left(0, E^{\star}\right)$ реализуется топологический случай 5, на луче $\left(E^{\star},+\infty\right)$ - топологический случай 1.

ДокАЗАТЕЛЬство следует из леммы 9 и рассмотрения знаков $\operatorname{Re} J(E)$ в нуле и на бесконечности луча $(0,+\infty)$.

СлеДСТВИЕ 2. Топологический случай 4 может реализоваться только на кривых $\eta=\eta_{0} u \bar{\eta}=\eta_{-1}$. 
Остается только проверить, какой случай реализуется на каждой из четырех связных компонент множества $(\eta \cup \bar{\eta}) \backslash\left\{E^{\star}\right\}$.

Лемма 10. Случай 4 реализуется на интервале кривой $\eta(\bar{\eta})$, лежащем между точкой $E^{\star}$ и точкой $i$ (соответственно $\left.-i\right)$. На оставиихся компонентах связности кривых $\eta$ и $\bar{\eta}$ реализуется топологический случай 3 .

ДокАЗАтЕльство аналогично доказательству следствия 1, оно следует из рассмотрения знаков $\operatorname{Re} \xi(0,2 \pi)$ на каждой компоненте связности.

Теорема 3. Топологический случай 1 реализуется на луче $\left(E^{\star},+\infty\right)$. Случай 4 на интервале кривой $\eta$, соединяющем точки $i$ и $E^{\star}$ (при этом в графе Стокса конечной линией Стокса соединены точки $z_{-}$и $\left.z_{+}\right)$, и на интервале кривой $\bar{\eta}$, соединяющем точки $-i$ и $E^{\star}$ (при этом в графе Стокса конечной линией Стокса соединены точки $\left.z_{-} u z_{+}-2 \pi\right)$. Случай 2 реализуется в точке $E^{\star}$. В остальных точках полуполосы $[0,+\infty)+i[-1,1]$, кроме точек $\pm i$, реализуются случаи 3 и 5 .

ДокАЗАТЕЛЬСТво проводится объединением лемм 6 и 10 и следствий 1 и 2.

\section{6. МАТРИЦА МОНОДРОМИИ И УСЛОВИЕ НА СПЕКТР}

Наличие нетривиального периодического решения уравнения $D \varphi-E \varphi=0$ эквивалентно наличию собственной функции $\psi_{0}$ с собственным значением 1 оператора монодромии $\mathfrak{M}$ (оператора сдвига решений на период). Пространство $L$ peшений уравнения $D \varphi-E \varphi=0$ двумерное, поэтому вместо оператора монодромии $\mathfrak{M}$ можно рассматривать матрицу монодромии (размера $2 \times 2$ ), являющуюся его координатной матрицей в некотором базисе пространства $L$. Тогда наличие собственной функции $\varphi_{0}$ с собственным значением 1 у оператора монодромии эквивалентно наличию собственного вектора с собственным значением 1 у матрицы монодромии $M$. Этот собственный вектор оператора монодромии является вектором координат функции $\psi_{0}$ в том же базисе пространства $L$, в котором строилась матрица $M$, т.е. $\operatorname{det}\left(M-\mathrm{id}_{2 \times 2}\right)=0$. Так как $\operatorname{det}\left(M-\operatorname{id}_{2 \times 2}\right)=\operatorname{det} M-\operatorname{tr} M+1$, то мы получаем условие $\operatorname{tr} M=\operatorname{det} M+1$ на матрицу монодромии, эквивалентное условию принадлежности $E$ дискретному спектру оператора $\mathfrak{D}$.

Для того чтобы вычислить матрицу монодромии, достаточно построить пару фундаментальных решений $\psi_{1}, \psi_{2}$ в некоторой связной области $G$, включающей в себя одновременно некоторое непустое открытое множество $U$ и множество $U+T$. Тогда при $z \in U$ имеем

$$
\left(\begin{array}{c}
\psi_{1}(z+T) \\
\psi_{2}(z+T)
\end{array}\right)=\left(\begin{array}{c}
\psi_{1}^{T}(z) \\
\psi_{2}^{T}(z)
\end{array}\right)=M \times\left(\begin{array}{c}
\psi_{1}(z) \\
\psi_{2}(z)
\end{array}\right),
$$

откуда матрица монодромии находится однозначно.

Фундаментальные решения $\psi_{1}$ и $\psi_{2}$ будем строить методом ВКБ-приближения (при этом и возникнут асимптотики для $h \rightarrow 0+0$ ). Напомним, что

$$
\xi\left(z_{0}, z\right)=\int_{z_{0}}^{z} \sqrt{i V(w)-E} d w
$$


где в качестве $z_{0}$ берется одна из точек поворота. Максимальные области однолистности функции $\xi$, содержащие точку $z_{0}$ на своей границе и ограниченные линиями Стокса, называются каноническими. В канонической области канонической парой фундаментальных решений является решение

$$
\begin{aligned}
& \psi_{1}(z)=(i V(z)-E)^{-1 / 4} e^{\xi\left(z_{0}, z\right) / h}(1+O(h)), \\
& \psi_{2}(z)=(i V(z)-E)^{-1 / 4} e^{-\xi\left(z_{0}, z\right) / h}(1+O(h)) .
\end{aligned}
$$

Если есть пересекающиеся канонические области $K$ и $K^{\circ}$, то в них соотношения (6) задают канонические пары $\left(\psi_{1}, \psi_{2}\right)$ и $\left(\psi_{1}^{\circ}, \psi_{2}^{\circ}\right)$. В пересечении $K \cap K^{\circ}$ определены обе эти пары. Так как $\left\langle\psi_{1}, \psi_{2}\right\rangle=L=\left\langle\psi_{1}^{\circ}, \psi_{2}^{\circ}\right\rangle$, то пара $\left(\psi_{1}^{\circ}, \psi_{2}^{\circ}\right)$ выражается через пару $\left(\psi_{1}, \psi_{2}\right)$ с помощью некоторой невырожденной комплексной матрицы размера $2 \times 2$. Если обозначить ее как $\Omega\left(K, K^{\circ}\right) \in M_{2 \times 2}(\mathbb{C})$, то

$$
\left(\begin{array}{c}
\psi_{1}^{\circ} \\
\psi_{2}^{\circ}
\end{array}\right)=\Omega\left(K, K^{\circ}\right) \times\left(\begin{array}{l}
\psi_{1} \\
\psi_{2}
\end{array}\right) .
$$

Матрица $\Omega\left(K, K^{\circ}\right)$ называется матрицей перехода из $K$ в $K^{\circ}$. Равенство $(7)$ дает возможность продолжить фундаментальную пару решений $\left(\psi_{1}, \psi_{2}\right)$ в область $K^{\circ}$. Аналогично с помощью матрицы перехода $\Omega\left(K^{\circ}, K\right)=\left(\Omega\left(K, K^{\circ}\right)\right)^{-1}$ фундаментальную пару решений $\left(\psi_{1}^{\circ}, \psi_{2}^{\circ}\right)$ можно продолжить в область $K$. Формулы для $\Omega\left(K, K^{\circ}\right)$ можно найти, например, в работах [8], [11]. В случае, когда $K$ и $K^{\circ}$ не пересекаются, если есть (конечная) цепочка канонических областей, объединение которых является связным и содержит $K$ и $K^{\circ}$, можно построить $\Omega\left(K, K^{\circ}\right)$ как произведение промежуточных матриц перехода.

Если бы области $U$ и $U+T$ вместе включались в некоторую каноническую область, то представления (6) было бы достаточно для вычисления матрицы монодромии $M$. Однако в нашем случае такой канонической области нет, и для того чтобы построить матрицу $M$, необходимо продолжить решения в некоторую нестягиваемую на $\mathbb{C} / T \mathbb{Z}$ область $G$. Для этого воспользуемся матрицами перехода, которые позволяют продолжить пару $\left(\psi_{1}, \psi_{2}\right)$ в другие канонические области. Будем делать это до тех пор, пока область определения пары решений $\left(\psi_{1}, \psi_{2}\right)$ не станет нестягиваемой на $\mathbb{C} / T \mathbb{Z}$. Тогда можно будет вычислить матрицу монодромии $M$, причем она будет выражаться через матрицы перехода.

Пусть в канонической области $K$ выбрана некоторая фундаментальная пара решений $\left(\psi_{1}(z), \psi_{2}(z)\right)$. Тогда в канонической области $K-T$ автоматически выбрана фундаментальная пара решений $\left(\psi_{1}^{T}(z), \psi_{2}^{T}(z)\right)$, т.е. если в области $K$ задана фундаментальная система решений $\left(\psi_{1}(z), \psi_{2}(z)\right)$, то в области $K-T$ автоматически задана система $\left(\psi_{1}^{T}(z), \psi_{2}^{T}(z)\right)$ :

$$
\left(\psi_{1}^{T}(z), \psi_{2}^{T}(z)\right)=\left(\psi_{1}(z+T), \psi_{2}(z+T)\right), \quad z \in K-T, \quad z+T \in K .
$$

С другой стороны, систему решений $\left(\psi_{1}(z), \psi_{2}(z)\right)$ можно продолжить в область $K-T$ (возможно, не напрямую, а через другие канонические области) с помощью 
матрицы перехода $\Omega$ (которая будет произведением матриц промежуточных переходов). Тогда в области $K-T$ будут определены две системы решений $\left(\psi_{1}^{T}(z), \psi_{2}^{T}(z)\right)$ и $\left(\psi_{1}(z), \psi_{2}(z)\right)$. Они будут отличаться друг от друга умножением слева на некоторую матрицу размера $2 \times 2$. Это и есть матрица монодромии.

Остается только выразить матрицу монодромии $M$ через матрицу перехода $\Omega$. Будем считать, что во всех последующих формулах $z \in K, z_{0}$ - точка поворота, $z_{0} \in \partial K$. Кроме того, введем следующие обозначения: $\left(\psi_{1}, \psi_{2}\right)$ - каноническая система решений в области $K,\left(\psi_{1}^{\circ}, \psi_{2}^{\circ}\right)$ - каноническая система решений в $K-T$, $\left(\psi_{1}^{T}, \psi_{2}^{T}\right)$ - фундаментальная (но не обязательно каноническая) система решений в $K-T$, получающаяся из $\left(\psi_{1}, \psi_{2}\right)$ сдвигом аргумента на период:

$$
\left(\psi_{1}^{T}(z-T), \psi_{2}^{T}(z-T)\right)=\left(\psi_{1}(z), \psi_{2}(z)\right), \quad z \in K
$$

В канонической области $K$ каноническая фундаментальная система решений $\left(\psi_{1}, \psi_{2}\right)$ задается формулами (6), которые в канонической области $K-T$ принимают вид

$$
\left(\begin{array}{c}
\psi_{1}^{\circ}(z-T) \\
\psi_{2}^{\circ}(z-T)
\end{array}\right)=(V(z-T)-E)^{-1 / 4}\left(\begin{array}{c}
e^{\xi\left(z_{0}-T, z-T\right) / h} \\
e^{-\xi\left(z_{0}-T, z-T\right) / h}
\end{array}\right), \quad z-T \in K-T .
$$

Так как функция $V$ периодична с периодом $T$, то $V(z-T)=V(z)$. Кроме того, снова по периодичности, $\xi\left(z_{0}-T, z-T\right)=\xi\left(z_{0}, z\right)$. С учетом этого имеем

$$
\left(\begin{array}{c}
\psi_{1}^{\circ}(z-T) \\
\psi_{2}^{\circ}(z-T)
\end{array}\right)=(V(z)-E)^{-1 / 4}\left(\begin{array}{c}
e^{\xi\left(z_{0}, z\right) / h} \\
e^{-\xi\left(z_{0}, z\right) / h}
\end{array}\right), \quad z-T \in K-T .
$$

Теперь выпишем $\left(\psi_{1}^{T}, \psi_{2}^{T}\right)$ :

$$
\left(\begin{array}{c}
\psi_{1}^{T}(z-T) \\
\psi_{2}^{T}(z-T)
\end{array}\right)=\left(\begin{array}{c}
\psi_{1}(z) \\
\psi_{2}(z)
\end{array}\right)=(V(z)-E)^{-1 / 4}\left(\begin{array}{c}
e^{\xi\left(z_{0}, z\right) / h} \\
e^{-\xi\left(z_{0}, z\right) / h}
\end{array}\right), \quad z-T \in K-T .
$$

Как нетрудно видеть, сравнивая (8) и (9), в области $K-T$ каноническая система решений $\left(\psi_{1}^{\circ}(z-T), \psi_{2}^{\circ}(z-T)\right)$ совпадает с фундаментальной $\left(\psi_{1}^{T}(z-T), \psi_{2}^{T}(z-T)\right)$.

Вспоминая выражения (7) и (5) и учитывая, что в канонической области $K-T$ имеем совпадение $\left(\psi_{1}^{\circ}, \psi_{2}^{\circ}\right)=\left(\psi_{1}^{T}, \psi_{2}^{T}\right)$, получаем, что матрица монодромии выражается через матрицу перехода тривиально: $M=\Omega$. Значит, условие принадлежности $E$ дискретному спектру оператора $\mathfrak{D}$ будет выражаться через матрицу перехода $\Omega$ так же, как оно выражается через матрицу монодромии:

$$
\operatorname{tr} \Omega=\operatorname{det} \Omega+1
$$

\section{7. АСИМПТОТИКА СПЕКТРА}

В этом разделе мы будем полагать, что $\operatorname{Im} z_{+} \in[0, \pi]$, это соответствует случаю $\operatorname{Im} E \geqslant 0$ на $E$-плоскости. Именно в этом предположении мы будем рисовать графы Стокса и рассчитывать условия на асимптотику. Как мы знаем (см. лемму 1), 
спектр симметричен относительно оси $\operatorname{Re} E$, и ограничение $\operatorname{Im} E \geqslant 0$ не является существенным. Нам придется только учесть, что полученное ниже условие (12) на $E$ имеет симметричный вариант, получающийся заменой $E$ на $\bar{E}$.

Топологический случай 1. Введем обозначения: $\gamma_{1}$ - линия Стокса, выходящая из точки $z_{+}$вертикально вверх; $\gamma_{2}$ - линия Стокса, соединяющая $z_{+}$с $z_{+}^{\prime}=$ $z_{+}+T$; а $\gamma_{1}^{\prime}=\gamma_{1}+T$ (см. рис. 6$)$.

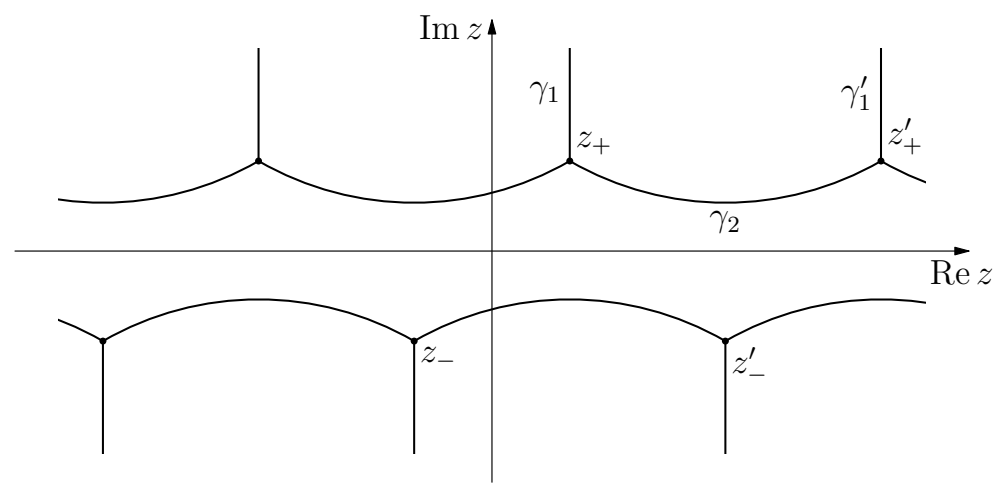

Рис. 6

Каждую из линий Стокса $\gamma_{1}, \gamma_{2}$ и $\gamma_{1}^{\prime}=\gamma_{1}+T$ можно включить в соответствующую каноническую область $K_{1}, K_{2}$ и $K_{1}^{\prime}=K_{1}+T$. Тогда, для того чтобы продолжить систему решений из области $K_{1}$ в область $K_{1}^{\prime}=K_{1}+T$, необходимо построить матрицы перехода $\Omega_{k}, k \in\{1,2,3\}$ :

$$
\left(\gamma_{1}, z_{+}, K_{1}\right) \stackrel{\Omega_{1}}{\longrightarrow}\left(\gamma_{2}, z_{+}, K_{2}\right) \stackrel{\Omega_{2}}{\longrightarrow}\left(\gamma_{2}, z_{+}^{\prime}, K_{2}\right) \stackrel{\Omega_{3}}{\longrightarrow}\left(\gamma_{1}^{\prime}, z_{+}^{\prime}, K_{1}^{\prime}\right)
$$

Переход от системы решений в области $K_{1}$ к системе решений в области $K_{1}^{\prime}$ будет задаваться произведением этих матриц $\Omega=\Omega_{3} \Omega_{2} \Omega_{1}$. Матрицы $\Omega_{1}$ и $\Omega_{3}$ имеют следующий вид (см. [11]):

$$
e^{-i \pi / 6}\left(\begin{array}{cc}
0 & 1 \\
1+O(h) & i(1+O(h))
\end{array}\right)
$$

а оставшаяся матрица побочно-диагональна:

$$
\begin{gathered}
\Omega_{2}=e^{i \tau_{0}}\left(\begin{array}{cc}
0 & e^{\tau_{1} / h} \\
e^{-\tau_{1} / h} & 0
\end{array}\right), \quad \tau_{1}=\xi\left(z_{+}, z_{+}^{\prime}\right), \quad \operatorname{Im} \tau_{1}>0, \\
\tau_{0}=\lim _{z \rightarrow z_{+}, z \in \gamma_{2}} \arg (V(z)-E)^{-1 / 4}-\lim _{z \rightarrow z_{+}^{\prime}, z \in \gamma_{2}} \arg (V(z)-E)^{-1 / 4}=-\frac{\pi}{6} .
\end{gathered}
$$

Таким образом, получаем

$$
\Omega=e^{-i \pi / 2}\left(\begin{array}{cc}
0 & e^{-\tau_{1} / h} \\
e^{\tau_{1} / h}(1+O(h)) & i\left(e^{-\tau_{1} / h}+e^{\tau_{1} / h}\right)(1+O(h))+e^{\tau_{1} / h} O(h)
\end{array}\right)
$$


т.е. $\operatorname{det} \Omega=1+O(h)$, а условие (10) принимает вид $\cos \left(\tau_{1} /(i h)\right)=1+O(h)$, что дает условие на величину интеграла $\tau_{1}$ :

$$
\frac{\tau_{1}}{h}=\frac{1}{h} \int_{z_{+}}^{z_{+}+T} \sqrt{i \cos z-E} d z \in i 2 \pi \mathbb{Z}(1+O(h)) .
$$

Как мы помним, действительная часть этого условия в нашем случае выполняется автоматически, так как

$$
\operatorname{Re} \int_{z_{+}}^{z_{+}+T} \sqrt{i V(z)-E} d z=\operatorname{Re} \int_{0}^{T} \sqrt{i V(x)-E} d x=0 .
$$

Мнимая же его часть дает условие принадлежности точки $E$ дискретному спектру оператора $\mathfrak{D}$ :

$$
\frac{1}{h} \operatorname{Im} \int_{0}^{T} \sqrt{i V(x)-E} d x \in 2 \pi \mathbb{Z}+O(h) .
$$

ЗАмечАниЕ 4. При достаточно малом $h$, как только окрестности различных точек $E$, удовлетворяющих условию $\frac{1}{h} \operatorname{Im} \int_{0}^{T} \sqrt{i V(x)-E} d x \in 2 \pi \mathbb{Z}$, перестают пересекаться, в окрестностях этих точек имеется ровно по одной точке спектра. Тогда с учетом леммы 1 получаем, что точки спектра, соответствующие этому топологическому случаю, лежат на оси $\operatorname{Re} E$ точно.

Топологический случай 5. Рассмотрим случай общего положения, т.е. случай, когда все линии Стокса уходят в бесконечность и ограниченных линий Стокса

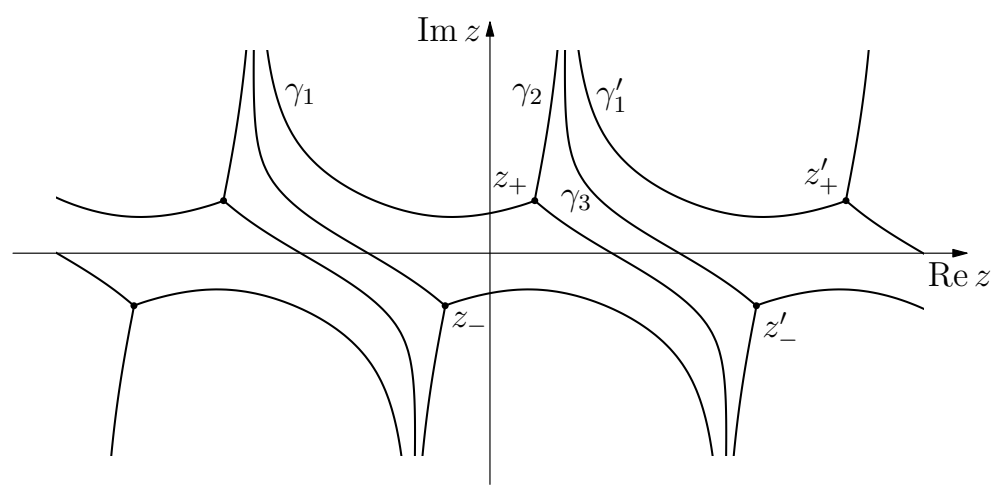

Рис. 7

нет. Картина линий Стокса для этого случая изображена на рис. 7. Введем обозначения: $\gamma_{1}, \gamma_{2}$ - линии Стокса, выходящие из точки поворота $z_{+}$и уходящие в $+i \infty$, a $\gamma_{3}$ - линия Стокса, выходящая из точки $z_{-}^{\prime}=z_{-}+T$ и также уходящая в $+i \infty$. Пусть, кроме того, $\gamma_{1}^{\prime}=\gamma_{1}+T$ и $z_{+}^{\prime}=z_{+}+T$. Каждую из линий Стокса $\gamma_{1}, \gamma_{2}, \gamma_{3}$ и $\gamma_{1}^{\prime}=\gamma_{1}+T$ можно включить в соответствующую каноническую область $K_{1}, K_{2}, K_{3}$ 
и $K_{1}^{\prime}=K_{1}+T$. Тогда для того чтобы продолжить систему решений из области $K_{1}$ в область $K_{1}^{\prime}=K_{1}+T$, необходимо построить матрицы перехода $\Omega_{k}, k \in\{1,2,3\}$ :

$$
\left(\gamma_{1}, z_{+}, K_{1}\right) \stackrel{\Omega_{1}}{\longrightarrow}\left(\gamma_{2}, z_{+}, K_{2}\right) \stackrel{\Omega_{2}}{\longrightarrow}\left(\gamma_{3}, z_{-}^{\prime}, K_{3}\right) \stackrel{\Omega_{3}}{\longrightarrow}\left(\gamma_{1}^{\prime}, z_{+}^{\prime}, K_{1}^{\prime}\right) .
$$

Переход от системы решений в области $K_{1}$ к системе решений в области $K_{1}^{\prime}$ будет задаваться произведением этих матриц $\Omega=\Omega_{3} \Omega_{2} \Omega_{1}$. Матрицы $\Omega_{k}$ имеют следующий вид (см. [11]):

$$
\begin{aligned}
\Omega_{1} & =e^{-i \pi / 6}\left(\begin{array}{cc}
0 & 1 \\
1+O(h) & i+O(h)
\end{array}\right), \\
\Omega_{2} & =e^{i \tau_{0}}\left(\begin{array}{cc}
e^{-\tau_{1} / h} & 0 \\
0 & e^{\tau_{1} / h}
\end{array}\right), \quad \tau_{1}=\xi\left(z_{+}, z_{-}^{\prime}\right), \quad \operatorname{Re} \tau_{1}>0, \\
\tau_{0} & =\lim _{z \rightarrow z_{+}, z \in \gamma_{2}} \arg (V(z)-E)^{-1 / 4}-\lim _{z \rightarrow z_{-}^{\prime}, z \in \gamma_{3}} \arg (V(z)-E)^{-1 / 4}, \\
\Omega_{3} & =e^{i \tau_{0}^{\prime}}\left(\begin{array}{cc}
e^{-\tau_{1}^{\prime} / h} & 0 \\
0 & e^{\tau_{1}^{\prime} / h}
\end{array}\right), \quad \tau_{1}^{\prime}=\xi\left(z_{-}^{\prime}, z_{+}^{\prime}\right), \quad \operatorname{Re} \tau_{1}^{\prime}>0, \\
\tau_{0}^{\prime} & =\lim _{z \rightarrow z_{-}^{\prime}, z \in \gamma_{3}} \arg (V(z)-E)^{-1 / 4}-\lim _{z \rightarrow z_{+}^{\prime}, z \in \gamma_{1}^{\prime}} \arg (V(z)-E)^{-1 / 4} .
\end{aligned}
$$

Таким образом, получаем

$$
\Omega=e^{i\left(\tau_{0}+\tau_{0}^{\prime}-\pi / 6\right)}\left(\begin{array}{cc}
0 & e^{-\left(\tau_{1}+\tau_{1}^{\prime}\right) / h} \\
e^{\left(\tau_{1}+\tau_{1}^{\prime}\right) / h}(1+O(h)) & i e^{\left(\tau_{1}+\tau_{1}^{\prime}\right) / h}(1+O(h))
\end{array}\right) .
$$

Для этой матрицы $\operatorname{det} \Omega=-e^{2 i\left(\tau_{0}+\tau_{0}^{\prime}-\pi / 6\right)}+O(h)$, откуда $\tau_{0}+\tau_{0}^{\prime}-\pi / 6=\pi / 2+\pi k . \mathrm{A}$ ее след $\operatorname{tr} \Omega=(-1)^{k+1} e^{\left(\tau_{1}+\tau_{1}^{\prime}\right) / h}(1+O(h))$. Нам подходит только $\tau_{0}+\tau_{0}^{\prime}-\pi / 6=\pi / 2+$ $2 \pi k$, при этом условие $(10)$ преобразуется в $\operatorname{tr} \Omega=e^{\left(\tau_{1}+\tau_{1}^{\prime}\right) / h}(1+O(h))=2+O(h)$. Так как $\operatorname{Re}\left(\tau_{1}+\tau_{1}^{\prime}\right)>0$, то $|\operatorname{tr} \Omega| \rightarrow+\infty$ при $h \rightarrow 0+0$, т.е. это условие асимптотически не выполняется. Другими словами, этот случай ничего не вносит в асимптотику спектра оператора $\mathfrak{D}$.

Топологический случай 3. Этот случай рассматривается аналогично предыдущему; он также ничего не вносит в асимптотику спектра оператора $\mathfrak{D}$.

Топологический случай 4. Граф Стокса для этого случая изображен на рис. 8. Вычисление матриц перехода, аналогичное проделанному выше для топологического случая 1 , приводит к следующему условию принадлежности точки $E$ спектру оператора $\mathfrak{D}$ :

$$
\frac{1}{h} \operatorname{Im} \int_{z_{-}}^{z_{+}} \sqrt{i \cos z-E} d z \in \frac{\pi}{2}+\pi \mathbb{Z}+O(h) .
$$

ЗАмечАниЕ 5. Как видно, как только $h$ становится настолько мало, что $O(h)$ окрестности разных точек $E$, удовлетворяющих условию (12), перестают пересекаться, в $O(h)$-окрестностях точек $E$, для которых выполняется условие (12), лежит ровно по одной точке спектра. 


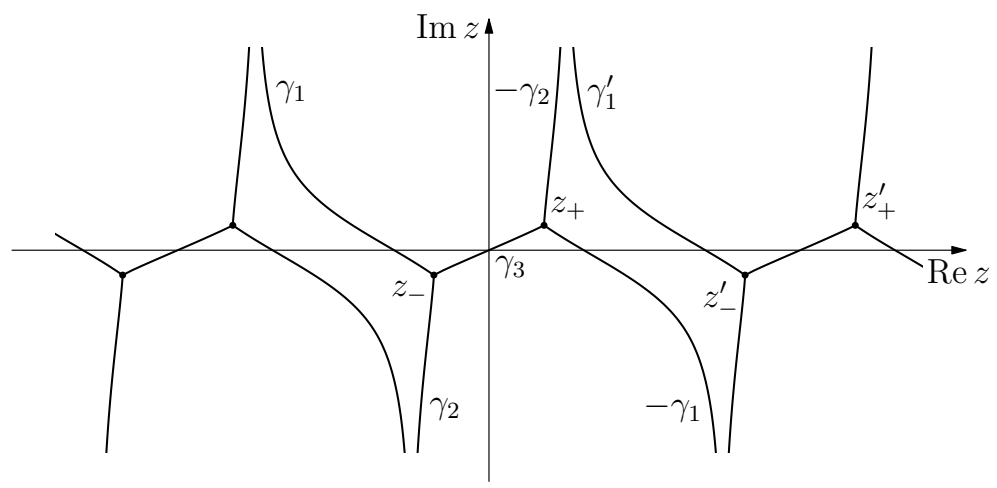

Рис. 8

Топологический случай 2. При $E=E^{\star}$ граф Стокса имеет вид, изображенный на рис. 9. В этом случае получаем следующее условие:

$$
\frac{1}{h} \operatorname{Im} \int_{z_{-}}^{z_{+}} \sqrt{i \cos z-E^{\star}} d z \in \frac{\pi}{6}+\pi \mathbb{Z}+O(h) .
$$

Оно разрешимо в некоторой окрестности точки $E^{\star}$ при любом $h$, т.е. $E^{\star}$ входит в предельный спектральный граф.

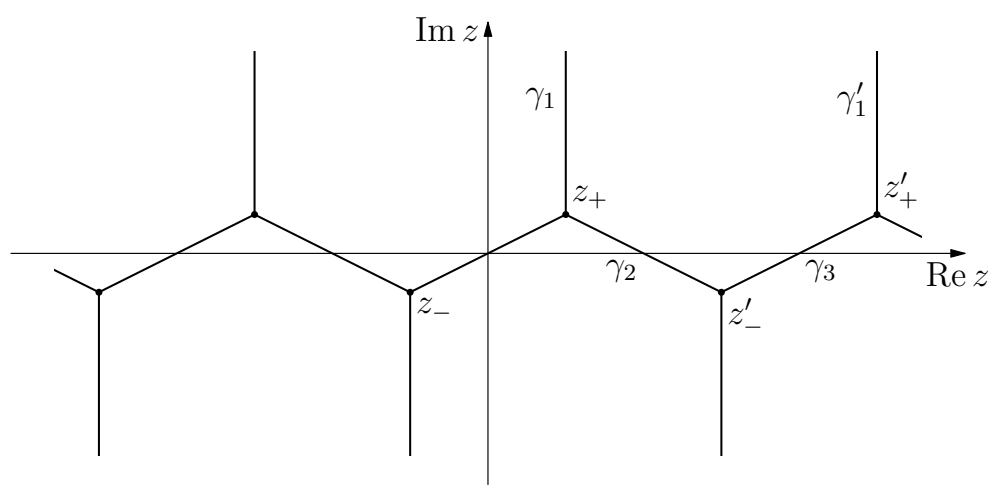

Рис. 9

Таким образом, нами доказана

Tеорема 4. В асимптотику спектра оператора $\mathfrak{D}$ вклад вносят только топологические случаи 1, 2 и 4. В случае 1 принадлежность точки E спектру определяется условием (11), причем все такие точки лежат на луче $\left(E^{\star},+\infty\right)$ точно. В случае 2 принадлежность точки $E$ определяется тем, удовлетворяет ли E или $\bar{E}$ условию (12). В окрестностях точек, удовлетворяющих асимптотикам (11) и (12) (возможно, кроме точки $\left.E^{\star}\right)$, лежит ровно по одной точке спектра (при достаточно мальх $h$ ). 


\section{8. ОСНОВНОЙ РЕЗУЛЬТАТ}

Теорема 5. Вне є-окрестностей точек $\pm i$ асимптотика дискретного спектра оператора $\mathfrak{D}$ при $i V(z)=i \cos z$ сосредоточена на трех кривых: луче $\left(E^{\star},+\infty\right)$, отрезке кривой $\eta$, соединяющем точку $i$ и точку $E^{\star}$, и отрезке кривой $\bar{\eta}$, соединяющем точку -i и точку $E^{\star}$. На луче $\left(E^{\star},+\infty\right)$ асимптотика дискретного спектра оператора $\mathfrak{D}$ выделяется условием $\operatorname{Im} \int_{0}^{2 \pi} \sqrt{i \cos x-E} d x \in h(2 \pi \mathbb{Z}+O(h))$, на отрезке кривой $\eta$ - условием (12), а на отрезке кривой $\bar{\eta}$ - сопряженным условием $\operatorname{Im} \int_{z_{-}(\bar{E})}^{z_{+}(\bar{E})} \sqrt{i \cos z-\bar{E}} d z \in h\left(\frac{\pi}{2}+\pi \mathbb{Z}+O(h)\right)$. Кроме того, справедливы следующие утверждения:

1) дискретный спектр оператора $\mathfrak{D}$ симметричен относительно оси $\operatorname{Re} E$;

2) в окрестностях точек, удовлетворяющих выписанным асимптотикам (возможно, кроме точки $\left.E^{\star}\right)$, лежит ровно по одной точке спектра;

3) на луче $\left[E^{\star},+\infty\right)$ дискретный спектр сосредоточен точно.

Для доказательства достаточно объединить результаты леммы 1 и теорем 2, 3 и 4.

Из теоремы 5 следует теорема 1.

ПРИЛОЖКЕНИЕ

Лемма 11. Для любого $a \in \mathbb{R}$ верно равенство

$$
\int_{z_{-}(E)}^{z_{+}(E)+a} \sqrt{i \cos z-E} d z=\int_{z_{-}(\bar{E})}^{z_{+}(\bar{E})-a-2 \pi} \sqrt{i \cos z-\bar{E}} d z .
$$

ДокАЗАтЕЛЬСтво. Из формулы (4) следует, что $\operatorname{Re} z_{ \pm}(\bar{E})= \pm \pi-\operatorname{Re} z_{ \pm}(E)$ и $\operatorname{Im} z_{ \pm}(\bar{E})=\operatorname{Im} z_{ \pm}(E)$, значит, $z_{ \pm}(\bar{E})= \pm \pi-\overline{z_{ \pm}(E)}$. С учетом этого и с помощью замены $z=-\bar{w}-\pi$ получаем требуемое.

Лемма 12. При $E \in \mathbb{C} \backslash i(-1,1)$ интеграл

$$
I(E)=\operatorname{Re} \int_{0}^{2 \pi} \sqrt{i \cos x-E} d x
$$

обращается в нуль тогда и только тогда, когда $E \in(0,+\infty)$.

ДокАЗАтЕЛЬСтво. Можно убедиться в том, что производная действительной части подынтегрального выражения по мнимой части $E$ отрицательна, а значит, интеграл монотонен по $\operatorname{Im} E$. Кроме того, легко показать, что при $E \in(0,+\infty)$ интеграл обращается в нуль.

Лемма 13. Уравнение

$$
J(E)=\operatorname{Re} \int_{z_{-}(E)}^{z_{+}(E)} \sqrt{i \cos z-E} d z=0
$$

на параметр $E \in(0,+\infty)$ имеет ровно одно решение $E=E^{\star}$, причем это решение $E^{\star} \in(0, \operatorname{sh}(\pi / 2))$.

3 Теоретическая и математическая физика, т. 148, № 2, 2006 г. 
ДокАзАтЕЛЬСтво. Легко показать, что $J(E)$ монотонна на $[0,+\infty)$ и принимает в нуле и точке $\operatorname{sh}(\pi / 2)$ значения разных знаков.

\section{Список литературы}

[1] И. Ц. Гохберг, М. Г. Крейн, Введение в теорию линейных несамосопряженных операторов, Наука, М., 1965.

[2] Я.Б. Зельдович, А. А. Рузмайкин, УФН, 152:2 (1987), 263-284.

[3] R. G. Drazin, W.H. Reid, Hydrodynamic Stability, Cambridge Univ. Press, Cambridge, 1981.

[4] В.П. Маслов, Теория возмущений и асимптотические методы, Изд-во МГУ, М., 1965; Асимптотические методы решения псевдодифференциальны уравнений, Наука, М., 1987.

[5] S. Yu. Dobrokhotov, V. N. Kolokoltsov, V. Martinez Olive, Sobretiro de Socieded Matematica Mexicana, 11 (1994), 81-89; С. Ю. Доброхотов, В.Н. Колокольцов, В. Мартинес Оливе, Матем. заметки, 58:2 (1995), 301-306.

[6] L. N. Trefethen, "Pseudospectra of linear operators", ISIAM 95: Proc. of the Third Int. Congress of Industrial and Applied Math. (Hamburg, Germany, July 3-7, 1995), Math. Res., 87, eds. K. Kirchgässner, O. Mahrenholtz, R. Mennicken, Akademie-Verlag, Berlin, 1996, 401-434; E. B. Davies, J. Oper. Theory, 43 (2000), 243-262.

[7] С. А. Степин, Матем. сб., 188 (1997), 129-146; С. А. Степин, А.А. Аржанов, УМН, 57:3 (2002), 161-162; А.А. Шкаликов, Матем. заметки, 62:6 (1997), 950-953.

[8] С. Н. Туманов, А. А. Шкаликов, Изв. РАН, Сер. матем., 66:4 (2002), 177-204.

[9] Ю.Н. Днестровский, Д. П. Костомаров, ДАН СССР, 152:1 (1963), 28-30.

[10] М. В. Федорюк, Изв. АН СССР, Сер. матем., 29:3 (1965), 645-656.

[11] М. А. Евграфов, М. В. Федорюк, УМH, 21:1 (1966), 3-50. 